\title{
Quantum-fluid description of the free-electron laser
}

\author{
R. Bonifacio, ${ }^{1}$ N. Piovella, ${ }^{2}$ G. R. M. Robb, ${ }^{3}$ and A. Serbeto ${ }^{4}$ \\ ${ }^{1}$ INFN Sezione di Milano, Via Celoria 16, Milano I-20133, Italy \\ ${ }^{2}$ Dipartimento di Fisica, Universita degli Studi di Milano, via Celoria 16, 20133 Milano, Italy \\ ${ }^{3}$ Scottish Universities Physics Alliance (SUPA), Department of Physics, University of Strathclyde, \\ 107 Rottenrow, Glasgow G4 ONG, United Kingdom \\ ${ }^{4}$ Instituto de Física, Universidade Federal Fluminense, 24210-340 Rio de Janeiro, Brazil
}

(Received 15 October 2008; published 15 January 2009)

\begin{abstract}
Using the Madelung transformation we show that in a quantum free-electron laser the beam obeys the equations of a quantum fluid in which the potential is the classical potential plus a quantum potential. The classical limit is shown explicitly.
\end{abstract}

DOI: 10.1103/PhysRevA.79.015801

PACS number(s): 42.50.Wk, 42.65.Sf

\section{INTRODUCTION}

In the quantum free-electron laser (FEL) model, the electron beam is described as a macroscopic matter wave [1-4]. When slippage due to the difference between the light and electron velocities is neglected, the electron-beam-wave interaction is described by the following equations for the dimensionless radiation amplitude $A(\bar{z})$ and the matter-wave field $\Psi(\theta, \bar{z})[5]$ :

$$
i \frac{\partial \Psi(\theta, \bar{z})}{\partial \bar{z}}=-\frac{1}{2 \bar{\rho}} \frac{\partial^{2}}{\partial \theta^{2}} \Psi(\theta, \bar{z})-i \bar{\rho}\left[A(\bar{z}) e^{i \theta}-\text { c.c. }\right] \Psi(\theta, \bar{z})
$$

$$
\frac{d A(\bar{z})}{d \bar{z}}=\int_{0}^{2 \pi} d \theta|\Psi(\theta, \bar{z})|^{2} e^{-i \theta}+i \delta A(\bar{z})
$$

The electron beam is therefore described by a Schrödinger equation for a matter-wave field $\Psi$ in a self-consistent pendulum potential proportional to $A$, where $|A|^{2}=|a|^{2} /(N \bar{\rho}),|a|^{2}$ is the average number of photons in the interaction volume $V$, and $|\Psi|^{2}$ is the space-time-dependent electron density, normalized to unity. In Eqs. (1) and (2) we have adopted the universal scaling used in the classical FEL theory [6-8], i.e., $\theta=\left(k+k_{w}\right) z-c k t$ is the electron phase, where $k_{w}=2 \pi / \lambda_{w}$ and $k=\omega / c=2 \pi / \lambda$ are the wiggler and radiation wave numbers, $\bar{z}=z / L_{g}$ is the dimensionless wiggler length, $L_{g}=\lambda_{w} / 4 \pi \rho$ is the gain length, $\rho=\gamma_{r}^{-1}\left(a_{w} / 4 c k_{w}\right)^{2 / 3}\left(e^{2} n / m \epsilon_{0}\right)^{1 / 3}$ is the classical FEL parameter, $\gamma_{r}=\sqrt{\left(\lambda / 2 \lambda_{w}\right)\left(1+a_{w}^{2}\right)}$ is the resonant energy in $m c^{2}$ units, $a_{w}$ is the wiggler parameter, and $n$ is the electron density. Finally, $\bar{p}=\left(\gamma-\gamma_{0}\right) / \rho \gamma_{0}$ is the dimensionless electron momentum and $\delta=\left(\gamma_{0}-\gamma_{r}\right) / \rho \gamma_{0}$ is the detuning parameter, where $\gamma_{0} \approx \gamma_{r}$ is the initial electron energy in $m c^{2}$ units.

Whereas the classical FEL equations in the above universal scaling do not contain any explicit parameter (see Ref. [8]), the quantum FEL equations (1) and (2) depend on the quantum FEL parameter

$$
\bar{\rho}=\left(\frac{m c \gamma_{r}}{\hbar k}\right) \rho .
$$

From the definition of $A$, it follows that $\bar{\rho}|A|^{2}=|a|^{2} / N$ is the average number of photons emitted per electron. Hence, since in the classical steady-state high-gain FEL $A$ reaches a maximum value of the order of unity, $\bar{\rho}$ represents the maximum number of photons emitted per electron, and the classical regime occurs for $\bar{\rho} \gg 1$. Note also that in Eq. (1) $\bar{\rho}$ appears as a "mass" term, so one expects a classical limit when the mass is large. As we shall see, when $\bar{\rho}<1$ the dynamical behavior of the system changes substantially from a classical to a quantum regime.

\section{QUANTUM FLUID DESCRIPTION}

We now perform a Madelung-like transformation $[9,10]$, writing the wave function as

$$
\Psi=R \exp (i \bar{\rho} S),
$$

which allows us to rewrite the Maxwell-Schrodinger equations, Eqs. (1) and (2), as a system of quantum fluid equations

$$
\begin{gathered}
\frac{\partial R}{\partial \bar{z}}=-\frac{\partial R}{\partial \theta} \frac{\partial S}{\partial \theta}-\frac{R}{2} \frac{\partial^{2} S}{\partial \theta^{2}}, \\
\frac{\partial S}{\partial \bar{z}}=-\frac{1}{2}\left(\frac{\partial S}{\partial \theta}\right)^{2}-V(\theta, \bar{z}), \\
\frac{d A}{d \bar{z}}=\int_{0}^{2 \pi} R^{2} e^{-i \theta} d \theta+i \delta A,
\end{gathered}
$$

where the potential $V$ in Eq. (5) is defined as the sum of a classical term and a quantum term, i.e.,

$$
V(\theta, \bar{z})=V_{C}+V_{Q}
$$

where

$$
V_{C}=-i\left(A e^{i \theta}-\text { c.c. }\right)
$$

is the classical component of the potential and 


$$
V_{Q}=-\frac{1}{2 \bar{\rho}^{2} R} \frac{\partial^{2} R}{\partial \theta^{2}}
$$

is the quantum component of the potential, which becomes negligible as $\bar{\rho} \rightarrow \infty$.

Defining fluid density and velocity variables

$$
n=R^{2}=|\Psi|^{2}, \quad u=\frac{\partial S}{\partial \theta},
$$

we can also rewrite Eqs. (4)-(6) in an alternative fluid form as

$$
\begin{gathered}
\frac{\partial n}{\partial \bar{z}}+\frac{\partial}{\partial \theta}(n u)=0, \\
\frac{\partial u}{\partial \bar{z}}+u \frac{\partial u}{\partial \theta}=-\frac{\partial V}{\partial \theta}, \\
\frac{d A}{d \bar{z}}=\int_{0}^{2 \pi} n e^{-i \theta} d \theta+i \delta A .
\end{gathered}
$$

It can be seen that Eq. (9) is a continuity equation and Eq. (10) is a Newton-like equation for a fluid. Note that when Eq. (9) is integrated with respect to $\theta$, then the normalization condition becomes

$$
\int_{0}^{2 \pi} n(\theta, \bar{z}) d \theta=1,
$$

which is satisfied if $n$ and $u$ are periodic functions of $\theta$ between 0 and $2 \pi$.

A straightforward calculation shows that Eqs. (9)-(11) admit two constants of motion,

$$
\langle\bar{p}\rangle+|A|^{2}=\mathcal{C}_{1}
$$

and

$$
\frac{\left\langle\bar{p}^{2}\right\rangle}{2}-i\left(A b^{*}-\text { c.c. }\right)-\delta|A|^{2}=\mathcal{C}_{2},
$$

where $\langle\bar{p}\rangle=\langle u\rangle=\int_{0}^{2 \pi} d \theta n u$ is the average momentum, $\left\langle\bar{p}^{2}\right\rangle$ $=\left\langle u^{2}+2 V_{Q}\right\rangle=\int_{0}^{2 \pi} d \theta n\left(u^{2}+2 V_{Q}\right)$ is the momentum variance, and

$$
b=\int_{0}^{2 \pi} n e^{-i \theta} d \theta
$$

is the bunching. These constants of motion are well known in the classical FEL model [8] and describe energy conservation and a gain-spread relation. Notice the quantum contribution to the momentum variance proportional to the average quantum potential.

\section{FOURIER EXPANSION AND LINEAR ANALYSIS}

If $R$ and $S$ are periodic functions of $\theta$, they can be expanded in a Fourier series:

$$
R(\theta, \bar{z})=\sum_{m} r_{m}(\bar{z}) e^{i m \theta},
$$

$$
S(\theta, \bar{z})=\sum_{n} s_{n}(\bar{z}) e^{i n \theta}
$$

with $r_{-m}=s_{m}^{*}$ and $s_{-m}=r_{m}^{*}$, since $R$ and $S$ are real variables. Multiplying Eq. (5) by $R$ and using (14) and (15) in Eqs. (4)-(6), we obtain

$$
\begin{aligned}
\sum_{m} r_{k-m} \frac{d s_{m}}{d \bar{z}}= & -\frac{1}{2} \sum_{m, n} n(n-m) r_{k-m} s_{n} s_{n-m}^{*} \\
& +i\left(A r_{k-1}-A^{*} r_{k+1}\right)-\frac{k^{2}}{2 \bar{\rho}^{2}} r_{k}, \\
\frac{d r_{k}}{d \bar{z}}= & \frac{1}{2} \sum_{m}\left(k^{2}-m^{2}\right) r_{m} s_{m-k}^{*}, \\
\frac{d A}{d \bar{z}}= & \sum_{m} r_{m} r_{m-1}^{*}+i \delta A .
\end{aligned}
$$

Equations (16)-(18) are our working equations which can be numerically solved, as will be shown elsewhere.

Equations (16)-(18) admit an equilibrium solution with no field $(A=0)$ and unbunched electron beam $(n=1 / 2 \pi$, i.e., $a_{n}=\delta_{n 0}$ and $\left.s_{n}=0\right)$. Linearizing Eqs. (16)-(18) around this equilibrium in the first order of the variables $A, r_{1}$, and $s_{1}$, we obtain

$$
\frac{d A}{d \bar{z}}=2 r_{1}+i \delta A
$$

$$
\frac{d r_{1}}{d \bar{z}}=\frac{s_{1}}{2},
$$

$$
\frac{d s_{1}}{d \bar{z}}=i A-\frac{1}{2 \bar{\rho}^{2}} r_{1} .
$$

Looking for solutions proportional to $\exp (i \lambda \bar{z})$, we obtain the well-known cubic equation of the quantum FEL [1],

$$
(\lambda-\delta)\left(\lambda^{2}-\frac{1}{4 \bar{\rho}^{2}}\right)+1=0,
$$

which reduces to the classical dispersion relation in the limit $\bar{\rho} \gg 1$.

\section{CONCLUSIONS}

It has been shown that the quantum FEL model can be rewritten in a form where the electron beam is described as a quantum fluid coupled to the electromagnetic field. The evolution of the quantum fluid is determined by a self-consistent potential which consists of a classical and a quantum contribution. In the limit where $\bar{\rho} \gg 1$, the quantum contribution to the potential becomes negligible and the force equation reduces to that of a Newtonian fluid. Using a Fourier expansion, linear stability analysis of these quantum fluid equations produced a dispersion relation identical to that derived from the Schrödinger equation. These results show that there are interesting connections between the quantum FEL and quantum plasma instabilities. 
[1] R. Bonifacio, M. M. Cola, N. Piovella, and G. R. M. Robb, Europhys. Lett. 69, 55 (2005).

[2] R. Bonifacio, N. Piovella, and G. R. M. Robb, Nucl. Instrum. Methods Phys. Res. A 543, 645 (2005).

[3] R. Bonifacio, N. Piovella, G. R. M. Robb, and A. Schiavi, Phys. Rev. ST Accel. Beams 9, 090701 (2006).

[4] A. Serbeto, J. T. Mendonca, K. H. Tsui, and R. Bonifacio, Phys. Plasmas 15, 013110 (2008).

[5] G. Preparata, Phys. Rev. A 38, 233 (1988).
[6] R. Bonifacio, C. Pellegrini, and L. Narducci, Opt. Commun. 50, 373 (1984).

[7] R. Bonifacio, L. De Salvo, P. Pierini, N. Piovella, and C. Pellegrini, Phys. Rev. Lett. 73, 70 (1994).

[8] R. Bonifacio, F. Casagrande, G. Cerchioni, L. De Salvo Souza, P. Pierini, and N. Piovella, Riv. Nuovo Cimento 13(9) (1990).

[9] A. Messiah, Quantum Mechanics (Wiley, London, 1966).

[10] E. Madelung, Z. Phys. 40, 332 (1926). 